\title{
Top Mixing
}

\section{F. del Aguila and J. A. Aguilar-Saavedra}

Departamento de Física Teórica y del Cosmos, Universidad de Granada, 18071 Granada, Spain E-mail: 'f aguila@ugr es' and 'aguilarj@ugr.es',

ABstract: We discuss the existing limits on top flavour-changing neutral couplings in models with new vector-like quarks. Large hadron and $e^{+} e^{-}$colliders can improve these bounds by more than one order of magnitude.

\section{Introduction}

The top quark is the heaviest fermion discovered up to now. Its mass $m_{t}=173.8 \pm 5.2 \mathrm{GeV}$ [i] $3.5 \times 10^{5}$ times the electron mass and makes more demanding the question of why the fermion mass hierarchy is so large. Not to say if we compare $m_{t}$ with the light neutrino masses. At present there is no compelling theoretical reason for such spreading. The Standard Model (SM) simply accommodates fermion masses and mixings. One is tempted, however, to relate the large value of the top mass, which is not very different from the electroweak symmetry breaking scale, with the mass generation mechanism. This and any other new physics are then expected to show up at the top first.

In contrast, top couplings are constrained rather poorly by present experimental data, especially the flavour-changing neutral (FCN) ones. In the SM there are no tree level FCN couplings and the effective ones induced at one loop are negligible. However, the top quark can have large tree level FCN couplings in models with extra vector-like quarks whose left-handed ( $\mathrm{LH})$ and right-handed $(\mathrm{RH})$ parts have the same transformation properties under $\mathrm{SU}(2)_{L}$. They are present in many extensions of the SM and are the only fermions whose mass can be banished to high energy.) Hence, it is theoretically important to constrain them experimentally. In Sections 2 and 3 we discuss the existing limits on these couplings in these simple and well-defined SM extensions [i=n. In Section
4 we comment on the bounds which can be obtained at large hadron and $e^{+} e^{-}$colliders. Existing limits will be eventually improved by at least

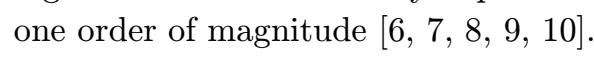

\section{Model-independent limits}

Direct limits on top FCN couplings can be derived from top decays. At present the most stringent bounds result from the non-observation of the decays $t \rightarrow Z q, t \rightarrow \gamma q, t \rightarrow g q$ at the Fermilab Tevatron. The neutral trilinear interactions between the top and a light quark $q$ are given by the Lagrangian

$$
\begin{aligned}
\mathcal{L}_{\mathrm{FCNC}}= & \bar{t}\left[\frac{g}{2 c_{W}} \gamma_{\mu}\left(X_{t q}^{L} P_{L}+X_{t q}^{R} P_{R}\right) Z^{\mu}\right. \\
& +\frac{g}{2 c_{W}}\left(\kappa_{t q}^{Z}-i \tilde{\kappa}_{t q}^{Z}\right) \frac{i \sigma_{\mu \nu} q^{\nu}}{m_{t}} Z^{\mu} \\
& +e\left(\kappa_{t q}^{\gamma}-i \tilde{\kappa}_{t q}^{\gamma}\right) \frac{i \sigma_{\mu \nu} q^{\nu}}{m_{t}} A^{\mu} \\
& \left.+g_{s}\left(\kappa_{t q}^{g}-i \tilde{\kappa}_{t q}^{g}\right) \frac{i \sigma_{\mu \nu} q^{\nu}}{m_{t}} T^{a} G^{a \mu}\right] q \\
& + \text { h.c. },
\end{aligned}
$$

with $P_{R, L}=\left(1 \pm \gamma_{5}\right) / 2$ and $T^{a}$ the Gell-Mann matrices satisfying $\operatorname{Tr}\left(T^{a} T^{b}\right)=\delta^{a b} / 2$. Tevatron bounds [1] $11,1,12]$

$$
\begin{aligned}
\operatorname{Br}(t \rightarrow Z q) & \leq 33 \% \\
\operatorname{Br}(t \rightarrow \gamma q) & \leq 3.2 \% \\
\operatorname{Br}(t \rightarrow g q) & \leq 15 \%
\end{aligned}
$$

translate into $\left|X_{t q}^{L}\right|,\left|X_{t q}^{R}\right| \leq 0.84,\left|\kappa_{t q}^{Z}\right|,\left|\tilde{\kappa}_{t q}^{Z}\right| \leq$ $0.78,\left|\kappa_{t q}^{\gamma}\right|,\left|\tilde{\kappa}_{t q}^{\gamma}\right| \leq 0.26,\left|\kappa_{t q}^{g}\right|,\left|\tilde{\kappa}_{t q}^{g}\right| \leq 0.15$. 
The effective couplings proportional to $\sigma_{\mu \nu}$ in Eq. $2.1 i$ are absent at tree level in renormalizable theories. Hence they are suppressed by one-loop factors $\sim \alpha / \pi$, and also by the GIM mechanism if there are no tree-level $\left(\gamma_{\mu}\right)$ FCN couplings. Thus we will neglect them in the following. However, SM extensions with vector-like quarks allow for large (tree-level) $\gamma_{\mu}$ FCN couplings. This is so because the addition of vectorlike quarks has little effect on radiative corrections. In particular, the new quarks decouple when their masses are taken to infinity. Although FCN couplings are constrained by precise electroweak data, indirect limits are at the mercy of possible cancellations between the top and other new particle contributions (see Ref. [i6] for an effective Lagrangian approach). It may be then convenient to derive these constraints in specific models. In the next Section we discuss these limits for SM extensions with vector-like fermions.

\section{Limits in extended models with vector-like quarks}

Let us consider a general SM extension with $N$ standard families, $n$ vector-like doublets and $n_{u}$ up and $n_{d}$ down vector-like singlets. (In practice, our limits will be saturated in the minimal extensions with $N=3$ and one extra doublet or singlet.) The quark content of this model is summarized in Table ${ }_{11}^{1}$. The weak eigenstates are then $q_{L, R}^{0}=\left(q_{L, R}^{(d)}, q_{L, R}^{(\vec{s})}\right)$, with $q_{L, R}^{(d)}$ doublets and $q_{L, R}^{(s)}$ singlets, and $q=u, d$.

$\begin{array}{lc}\text { LH doublets } & N+n \\ \text { RH doublets } & n \\ \text { up LH singlets } & n_{u} \\ \text { up RH singlets } & N+n_{u} \\ \text { down LH singlets } & n_{d} \\ \text { down RH singlets } & N+n_{d}\end{array}$

Table 1: Quark content of the model

Note that the total number of up-type quarks $\mathcal{N}_{u}=N+n+n_{u}$ and down-type quarks $\mathcal{N}_{d}=$ $N+n+n_{d}$ do not need to be equal in general. As in the SM, only isodoublets have $\mathrm{SU}(2)_{L}$ couplings, then the gauge neutral current La- grangian in the weak eigenstate basis can be written as

$$
\begin{aligned}
\mathcal{L}_{Z}= & -\frac{g}{2 c_{W}}\left(\bar{u}_{L}^{(d)} \gamma^{\mu} u_{L}^{(d)}+\bar{u}_{R}^{(d)} \gamma^{\mu} u_{R}^{(d)}\right. \\
& -\bar{d}_{L}^{(d)} \gamma^{\mu} d_{L}^{(d)}-\bar{d}_{R}^{(d)} \gamma^{\mu} d_{R}^{(d)} \\
& \left.-2 s_{W}^{2} J_{\mathrm{EM}}^{\mu}\right) Z_{\mu} .
\end{aligned}
$$

With this notation the only apparent difference with the SM Lagrangian is in the new RH currents. However, when we express this Lagrangian in the mass eigenstate basis the particular features of this model manifest. In this basis,

$$
\begin{aligned}
\mathcal{L}_{Z}= & -\frac{g}{2 c_{W}}\left(\bar{u}_{L} X^{u L} \gamma^{\mu} u_{L}+\bar{u}_{R} X^{u R} \gamma^{\mu} u_{R}\right. \\
& -\bar{d}_{L} X^{d L} \gamma^{\mu} d_{L}-\bar{d}_{R} X^{d R} \gamma^{\mu} d_{R} \\
& \left.-2 s_{W}^{2} J_{\mathrm{EM}}^{\mu}\right) Z_{\mu},
\end{aligned}
$$

where $u=(u, c, t, T, \ldots)$ and $d=(d, s, b, B, \ldots)$ are $\mathcal{N}_{u}$ and $\mathcal{N}_{d}$ dimensional vectors, respectively. The $X$ 's are matrices in flavour space. Apart from the new RH terms, the most important difference with the SM Lagrangian is that now the LH currents are not flavour diagonal, $i . e$. the $X$ 's are nondiagonal. To show this, let us write the unitary transformation between the mass and weak interaction eigenstates, $q_{L}^{0}=\mathcal{U}^{q L} q_{L}, q_{R}^{0}=$ $\mathcal{U}^{q R} q_{R}$, with $\mathcal{U}^{q L}$ and $\mathcal{U}^{q R} \mathcal{N}_{q} \times \mathcal{N}_{q}$ unitary matrices. Then,

$$
\begin{aligned}
X_{\alpha \beta}^{u L} & =\left(\mathcal{U}_{i \alpha}^{u L}\right)^{*} \mathcal{U}_{i \beta}^{u L}, \\
X_{\alpha \beta}^{u R} & =\left(\mathcal{U}_{j \alpha}^{u R}\right)^{*} \mathcal{U}_{j \beta}^{u R}, \\
X_{\sigma \tau}^{d L} & =\left(\mathcal{U}_{k \sigma}^{d L}\right)^{*} \mathcal{U}_{k \tau}^{d L}, \\
X_{\sigma \tau}^{d R} & =\left(\mathcal{U}_{l \sigma}^{d R}\right)^{*} \mathcal{U}_{l \tau}^{d R},
\end{aligned}
$$

where $(i, k)$ and $(j, l)$ sum over the left- and righthanded doublets, respectively, $\alpha, \beta=u, c, t, T, \ldots$ and $\sigma, \tau=d, s, b, B, \ldots$ In the SM, $\mathcal{U}^{q L}, \mathcal{U}^{q R}$ are $3 \times 3$ matrices and $i, k$ run from 1 to 3 , hence $X_{q q^{\prime}}^{u L, d L}=\delta_{q q^{\prime}}$ by unitarity for any mass eigenstates $q, q^{\prime}$. This is the well-known GIM mechanism. Besides, $X_{q q^{\prime}}^{u R, d R}=0$ for there are no $\mathrm{RH}$ doublets. In general, with $n>0$ and $N+n<$ $\mathcal{N}_{u}, \mathcal{N}_{d}$ the $X$ 's are products of submatrices with less rows than columns and thus they are nondiagonal in general.

At this point one would have to wonder about the constraints on the $X$ 's from precise electroweak data, and in particular from rare processes. However, from the apparently trivial Eqs. 3.3 we will 
find a set of inequalities which imply limits on the top couplings strong enough to fulfil all experimental data. To be definite, we work with $\mathcal{U}^{u L}$, and to simplify the notation, we write

$$
\mathcal{U}^{u L}=\left(\begin{array}{lllll}
a_{u} & a_{c} & a_{t} & \cdots & a_{\mathcal{N}_{u}} \\
b_{u} & b_{c} & b_{t} & \cdots & b_{\mathcal{N}_{u}}
\end{array}\right) \begin{aligned}
& \} N+n \\
& \} n_{u}
\end{aligned}
$$

with $a_{\alpha}, b_{\alpha}$ column vectors (remember that $N+$ $n$ is the number of up LH doublets and $n_{u}$ the number of up LH singlets). With this notation, orthogonality between columns of $\mathcal{U}^{u L}$ is written as $a_{\alpha} \cdot a_{\beta}+b_{\alpha} \cdot b_{\beta}=\delta_{\alpha \beta}$, and $X^{u L}$ can be written as $X_{\alpha \beta}^{u L}=a_{\alpha} \cdot a_{\beta}$ with the complex scalar product ' ' (in particular this shows that $\left|X_{\alpha \beta}^{u L}\right| \leq 1$ and $\left.X_{\alpha \alpha}^{u L} \geq 0\right)$. It is then easy to apply the Schwarz inequality to obtain

$$
\begin{aligned}
\left|X_{\alpha \beta}^{u L}\right|^{2} & =\left|a_{\alpha} \cdot a_{\beta}\right| \leq\left|a_{\alpha}\right|^{2}\left|a_{\beta}\right|^{2} \\
& =X_{\alpha \alpha}^{u L} X_{\beta \beta}^{u L},
\end{aligned}
$$

and for $\alpha \neq \beta$,

$$
\begin{aligned}
\left|X_{\alpha \beta}^{u L}\right|^{2} & =\left|a_{\alpha} \cdot a_{\beta}\right|=\left|b_{\alpha} \cdot b_{\beta}\right| \leq\left|b_{\alpha}\right|^{2}\left|b_{\beta}\right|^{2} \\
& =\left(1-\left|a_{\alpha}\right|^{2}\right)\left(1-\left|a_{\beta}\right|^{2}\right) \\
& =\left(1-X_{\alpha \alpha}^{u L}\right)\left(1-X_{\beta \beta}^{u L}\right) .
\end{aligned}
$$

The same can be done for down quarks and $\mathrm{RH}$ couplings, so we drop superscripts and write in complete generality

$$
\begin{aligned}
& \left|X_{q q^{\prime}}\right|^{2} \leq\left(1-X_{q q}\right)\left(1-X_{q^{\prime} q^{\prime}}\right), \\
& \left|X_{q q^{\prime}}\right|^{2} \leq X_{q q} X_{q^{\prime} q^{\prime}} .
\end{aligned}
$$

It is important to remark that these inequalities are valid for any number of generations $N$, doublets $n$ and singlets $n_{u}, n_{d}$. In particular, if $X_{q q}=0,1$, Eqs. 3.7 imply $X_{q q^{\prime}}=0$ independently of $X_{q^{\prime} q^{\prime}}$. In this way, Eqs. as the generalization of the GIM mechanism for models with vector-like quarks. In the case of the top quark, these equations allow to derive bounds on $X_{t u}, X_{t c}$, despite our ignorance on $X_{t t}$.

To use the inequalities in Eqs. to extract the values of the diagonal elements $X_{q q}$ from experimental data. From atomic par-

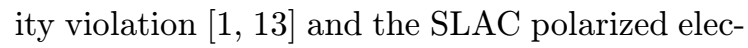
tron experiment [1] the diagonal $X$ elements of the $u$ and $d$ quarks in Table $\overline{2}_{1}^{1}$ can be extracted, whereas the measurement of $R_{b}, R_{c}, A_{\mathrm{FB}}^{0, b}, A_{\mathrm{FB}}^{0, c}$ at the CERN $e^{+} e^{-}$collider LEP and SLC [1] provides the diagonal $X$ elements of the $b$ and $c$ quarks (see Ref. [i5i] for details). We observe that the values of $X_{u u}^{R}, X_{d d}^{L}, X_{c c}^{R}$ and $X_{b b}^{R}$ in Table $\overline{2}_{1}^{\prime}$ are unphysical. This is worst for $X_{b b}^{R}$, which is $2 \sigma$ away from the physical region $[0,1]$, a direct consequence of the $2 \sigma$ discrepancy between the measured and the SM values of $A_{F B}^{0, b}$. It is then necessary a careful application of the inequalities. We define the $90 \%$ C. L. upper limit on $X_{q q^{\prime}}$ as the value $x$ such that the probability of finding $X_{q q^{\prime}} \leq x$ within the physical region is 0.9. With this definition and a Monte Carlo generator for the Gaussian distributions of $R_{b}, R_{c}$, $A_{F B}^{0, b}, A_{F B}^{0, c}$ (correlated) and $X_{u u}^{L, R}, X_{d d}^{L, R}$ (assuming no correlation) we obtain the bounds in Table 3. where we also quote existing limits in the lit-

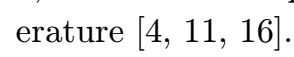

$$
\begin{aligned}
& X_{u u}^{L}=0.965 \pm 0.032 \\
& X_{u u}^{R}=-0.049 \pm 0.026 \\
& X_{d d}^{L}=1.035 \pm 0.022 \\
& X_{d d}^{R}=0.209_{-0.154}^{+0.096} \\
& X_{c c}^{L}=0.998 \pm 0.013 \\
& X_{c c}^{R}=-0.013 \pm 0.019 \\
& X_{b b}^{L}=0.996 \pm 0.005 \\
& X_{b b}^{R}=-0.039 \pm 0.018
\end{aligned}
$$

Table 2: Diagonal elements in Eq. ${ }_{3}^{-13} \cdot \overline{2}$

It is worth to emphasize that with Eqs. and precise electroweak data we can set limits on top couplings that $(i)$ are much stronger than present direct limits, and (ii) can be saturated in minimal SM extensions with one doublet or singlet and still fulfil all present experimental constraints [0-5in]

\section{Future limits}

Improvements on the determination of the top FCN couplings should come from large hadron and $e^{+} e^{-}$colliders. At these top factories the top FCN vertices manifest in top decays and in single top production. For $\sigma_{\mu \nu}$ vertices and large center of mass energies single top production is a more efficient way to look for FCN couplings because the extra $q^{\nu}$ factor in the vertex increases the large transverse momentum distributions im- 


\begin{tabular}{cccl} 
Coupling & $X^{L}$ & $X^{R}$ & Source \\
\hline$u c$ & $1.2 \times 10^{-3}$ & $1.2 \times 10^{-3}$ & $\delta m_{D}$ \\
& 0.033 & 0.019 & Inequalities \\
\hline$u t$ & 0.84 & 0.84 & $t \rightarrow u Z$ \\
& 0.28 & 0.14 & Inequalities \\
\hline$c t$ & 0.84 & 0.84 & $t \rightarrow c Z$ \\
& 0.14 & 0.16 & Inequalities \\
\hline$d s$ & $4.1 \times 10^{-5}$ & $4.1 \times 10^{-5}$ & $K^{+} \rightarrow \pi^{+} \nu \bar{\nu}$ \\
& 0.14 & 0.62 & Inequalities \\
\hline$d b$ & $1.1 \times 10^{-3}$ & $1.1 \times 10^{-3}$ & $\delta m_{B}$ \\
& 0.0081 & 0.062 & Inequalities \\
\hline$s b$ & $1.9 \times 10^{-3}$ & $1.9 \times 10^{-3}$ & $B^{0} \rightarrow \mu^{+} \mu^{-} X$ \\
& 0.076 & 0.12 & Inequalities
\end{tabular}

Table 3: Present bounds on nondiagonal elements in Eq. $13 . \overline{2}$

proving the signal to background ratios, whereas in top decays the energy available is typically smaller and the characteristic $q^{\nu}$ factor makes no appreciable difference. For definiteness, we restrict ourselves to renormalizable $Z t q$ couplings, which are the relevant ones in extended models with vector-like quarks. (The other couplings can be discussed in an analogous way.) $e^{+} e^{-}$ machines are a cleaner environment but less efficient top factories. LEP2 can improve present Tevatron limits at most by a factor of 3 [i $\overline{7}]$ and Tevatron Run II with a luminosity of $2 \mathrm{fb}^{-1}$ will improve LEP2 bounds by a factor of 2 [1 100.1$]$. Finally, LHC with a luminosity of $100 \mathrm{fb}^{-1}$ will improve the former bounds by a factor of 20 [1] 1 in]. Linear colliders will have to be consistent with the LHC limits but are not expected to improve them. However, if a sizeable coupling is found, linear colliders will be able to disentangle better the $\mathrm{P}$ and $\mathrm{CP}$ structure of the vertex.

\section{Acknowledgments}

We thank Ll. Ametller, G. C. Branco and R. Miquel for previous collaboration on this subject. F. A. thanks the organizers of the Summer Institute for their hospitality. This work was partially supported by CICYT under contract AEN961672 and by the Junta de Andalucía, FQM101.

\section{References}

[1] C. Caso et al., European Phys. Journal C3, 1 (1998)

[2] P. Langacker and D. London, Phys. Rev. D38, 886 (1988); D. London, in Precision Tests of the Standard Model, World Scientific, 1993; E. Nardi, E. Roulet and D. Tommasini, Phys. Rev. D46, 3040 (1992); see also J. L. Hewett and T. G. Rizzo, Phys. Rep. 183, 193 (1989)

[3] G. C. Branco and L. Lavoura, Nucl. Phys. B278, 738 (1986); R. Barbieri and L. J. Hall, Nucl. Phys. B319, 1 (1989); G. C. Branco, P. A. Parada and M. N. Rebelo, Phys. Rev. D52, 4217 (1995); F. del Aguila, J. A. AguilarSaavedra and G. C. Branco, Nucl. Phys. B510, 39 (1998); G. Barenboim, F. J. Botella, G. C. Branco and O. Vives, Phys. Lett. B422, 277 (1998)

[4] V. Barger, M. S. Berger and R. J. N. Phillips, Phys. Rev. D52, 1663 (1995)

[5] F. del Aguila, J. A. Aguilar-Saavedra and R. Miquel, Phys. Rev. Lett. 82, 1628 (1999)

[6] T. Han, R. D. Peccei and X. Zhang, Nucl. Phys. B454, 527 (1995)

[7] T. Han, K. Whisnant, B.-L. Young and X. Zhang, Phys. Rev. D55, 7241 (1997)

[8] T. Han, M. Hosch, K. Whisnant, B.-L. Young and X. Zhang, Phys. Rev. D58, 073008 (1998)

[9] J. L. Hewett, Int. J. Mod. Phys. A13, 2389 (1998); T. Han and J. L. Hewett, hepph/9811237, Phys. Rev. D (in press)

[10] F. del Aguila, J. A. Aguilar-Saavedra and Ll. Ametller, UG-FT-98/99, hep-ph/9906462 
[11] F. Abe et al., Phys. Rev. Lett. 80, 2525 (1998)

[12] T. Han, K. Whisnant, B.-L. Toung and X. Zhang, Phys. Lett. B385, 311 (1996)

[13] C. S. Wood et al., Science 275, 1759 (1997); see also S. C. Bennett and C. E. Wieman, Phys. Rev. Lett. 82, 2484 (1999)

[14] C. Y. Prescott et al., Phys. Lett. B84, 524 (1979)

[15] D. Karlen, plenary talk, ICHEP98, Vancouver, July 1998; see also D. Abbaneo et al., CERNPPE/97-154

[16] Y. Nir and D. Silverman, Phys. Rev. D42, 1477 (1990); D. Silverman, Phys. Rev. D58, 095006 (1998)

[17] DELPHI Collaboration, S. Andringa et al., DELPHI Report No. 98-70 CONF 138

[18] F. del Aguila and J. A. Aguilar-Saavedra, UGFT-100/99, in preparation 